Planetary Systems in the Universe - Observation, Formation and Evolution

Proceedings IAU Symposium No. 202, (C)2004 IAU

Alan Penny, Pawel Artymowicz, Anne-Marie Lagrange, \& Sara Russell, eds.

\title{
A Search for the Spectroscopic Signature of Hot Jupiters
}

\author{
P.W. Lucas \\ University of Hertfordshire, College Lane, Hatfield AL10 9AB, UK \\ P.F. Roche \\ University of Oxford, Astrohysics Dept,, 1 Keble Road, Oxford OX1 \\ 3RH, UK
}

\begin{abstract}
We describe a method by which hot extrasolar planets close to their parent star may be directly detected through the signature of water vapour or methane in their infrared spectra, lying on top of the spectrum of the parent star. Upper limits derived from low resolution $\mathrm{K}$ band spectra of several 'hot Jupiter' systems are presented. We find no water vapour to 3- $\sigma$ limits of between 1 part in 200 and 1 part in 600 for each star. Comparison with an initial model indicates that constraints can be set on the size, albedo, temperature and dust content of the planets.
\end{abstract}

\section{Introduction}

Elementary calculations indicate effective temperatures of $900-1600 \mathrm{~K}$ for the known short-period planet candidates, with orbital radii between 0.04 and 0.1 $\mathrm{au}$, owing to the intense insolation in that environment. Model atmosphere calculations have shown that the spectral energy distributions of substellar bodies bear little relation to the Planck function: so-called super-Planckian emission occurs at several near infrared wavelengths due to the many sources of molecular opacity $\left(\mathrm{H}_{2} \mathrm{O}, \mathrm{H}_{2}, \mathrm{CH}_{4}, \mathrm{H}^{-}\right.$etc. $)$and has been confirmed through spectroscopy of $\mathrm{T}$ dwarfs. Hence, at wavelengths of $2-4 \mu \mathrm{m}$ the stellar:companion flux ratio may be only a few hundred for the hotter sources.

If a sufficiently high signal to noise spectrum can be obtained, the planetary spectrum will be distinguishable by broad edges of absorption by $\mathrm{H}_{2} \mathrm{O}$ and also $\mathrm{CH}_{4}$ in the cooler sources. The stellar spectra are essentially featureless Planckian curves for F-type and early G-type stars. We have chosen to work at low spectral resolution, which makes it easier to obtain a high signal to noise ratio and removes the need to follow the orbital phase. Any candidate signal could be confirmed by observation in both the $\mathrm{K}$ and $\mathrm{L}$ wavebands.

\section{Method}

In order to obtain the highest signal to noise and remove the huge underlying stellar signal, we made back to back observations of each target star and several standards of identical spectral type at a very similar airmass and within a few degrees on the sky. We found that HIPPARCOS V-I and B-V colours, combined with parallaxes, were a reliable means of choosing standards of nearly identical 
standards. Observations were undertaken at the United Kingdom Infrared Telescope, with the spectrometer CGS4, in 1999 January, 1999 September and 1999 November. The key factor in obtaining a good division of object and standard was to ensure that the airmasses are as nearly identical as possible, and preferably $<1.05$. Post observation scaling of the airmasses was imperfect, probably because the telluric water column changes in an unpredictable way.

For each candidate system, every subset of data was divided by stellar standard spectra at similar airmass, using the IRAF function telluric to factor in the nominal airmass (not the water vapour column) and correct any offset in wavelength via a least squares fit.

For the $\mathrm{K}$ band data, a straight line was then fitted to the divided spectrum in the regions 2.03-2.10 $\mu \mathrm{m}$ (water) and 2.16-2.28 $\mu \mathrm{m}$ (continuum), excluding the $\mathrm{Br} \gamma$ residual at $2.166 \mu \mathrm{m}$. Water absorption would produce a difference in gradient between the two regions. If methane absorption were present at 2.16$2.28 \mu \mathrm{m}$ it would have the opposite slope to the water feature and therefore increase the size of any apparent molecular signal.

\section{Results}

No water or methane features were detected in any of the spectra at $\mathrm{K}$ band. We place upper limits on the strength of the water feature, using the mean deviation of the 'water' and 'continuum' slopes in multiple object/standard datasets for each system. Between two and five different standards were used for each system. The L band data were generally of slightly poorer quality in the region of the water edge $(3.45-3.5 \mu \mathrm{m})$ and have yet to be fully analysed.

We give upper limits represented as ratios of the stellar flux to the full depth of the expected water edge from 1.9-2.15 $\mu \mathrm{m}$. The quoted values are at $3 \times$ the mean deviation of slopes rather than 3- $\sigma$ since the more poorly matched object/standard pairs tend to inflate the usual r.m.s. standard deviation measure.

\begin{tabular}{lcccc} 
Source & Sp. Type & Orbital Radius (au) & M.sin(i) & Water Limit \\
\hline & & & & \\
Tau Boo A & F7V & 0.046 & $3.87 \mathrm{M}_{J}$ & $<1$ in 340 \\
51 Peg & G2V & 0.05 & $0.47 \mathrm{M}_{J}$ & $<1$ in 301 \\
HD 283750 & K2V & 0.025 & $50 \mathrm{M}_{J}$ & K-type: no result \\
$v$ And & F8V & 0.059 & $0.71 \mathrm{M}_{J}$ & $<1$ in 243 \\
HD 187123 & G5V & 0.042 & $0.52 \mathrm{M}_{J}$ & $<1$ in 565 \\
HD 209458 & G0V & 0.045 & $0.69 \mathrm{M}_{J}$ & $<1$ in 291
\end{tabular}

\section{Implications and Limits on Hot Planets}

These non-detections can be used to place limits on the properties of hot planets. Detectability is determined by Temperature $\left(\mathrm{T}_{e f f}\right)$, Radius and the prominence of the molecular absorption features.

Using the star+planet model with $\mathrm{T}_{e f f}=1600 \mathrm{~K}, \mathrm{R}_{P}=1.4 \mathrm{R}_{J} ; \mathrm{R}_{\text {Star }}=$ $1 \mathrm{R}_{\odot}$ and $\mathrm{T}_{\text {eff }}=5770 \mathrm{~K}$ for the star we would expect the depth of the $2 \mu \mathrm{m}$ water band to be 1 part in 800 . (The planetary radius is as measured in the transits of HD 209458b, eg. Charbonneau et al. 1999; Henry et al 2000) 


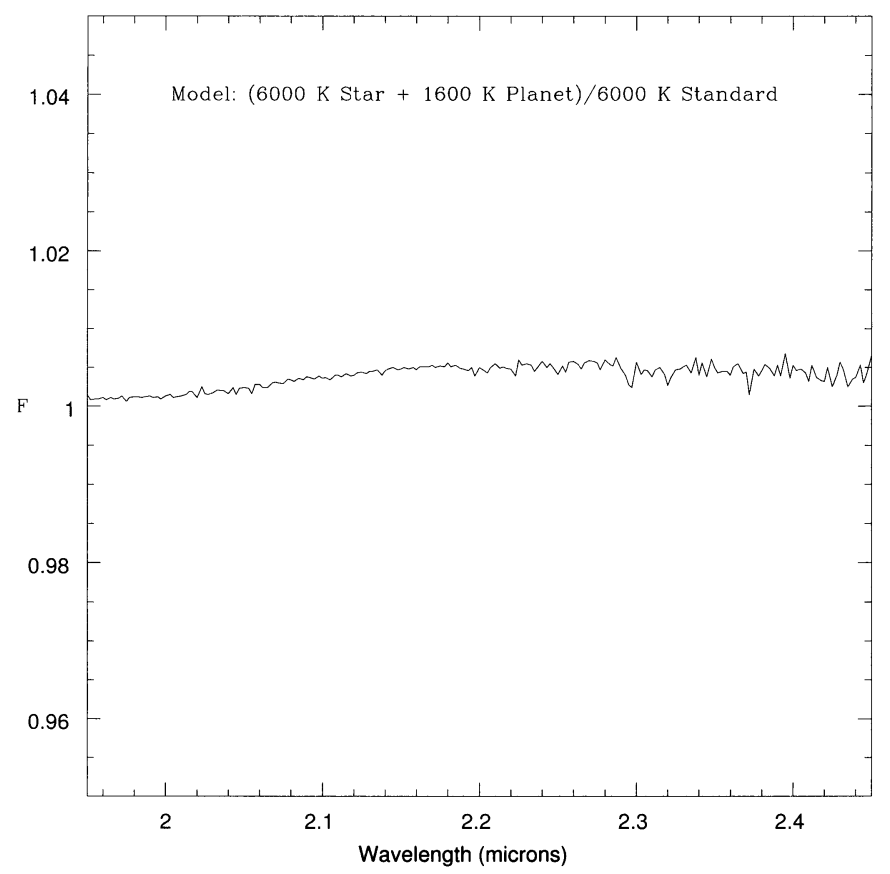

Figure 1. Preliminary model spectrum for a $1600 \mathrm{~K}$ planet, courtesy of Mark Marley and Didier Saumon of New Mexico State University and Vanderbildt University respectively. The water absorption edge from 1.95 to $2.15 \mu \mathrm{m}$ is apparent in the model but there is no sign of it in the data. We have added the planetary model to a stellar Black Body at $6000 \mathrm{~K}$, arbitrarily scaled so that the depth of the water absorption trough is 1 part in 290 of the peak flux.

Although we do not quite reach a sensitivity of 1 part in 800 , we can rule out the presence of objects with radii only slightly larger than $1.4 \mathrm{R}_{J}$, assuming that the $1600 \mathrm{~K}$ model is correct. For HD 187123 (the best case) we infer $\mathrm{R}_{P} \lesssim 1.67 \mathrm{R}_{J}$ for a $1600 \mathrm{~K}$ planet. For the other sources, the typical limit is $\mathrm{R}_{P} \lesssim 2.3 \mathrm{R}_{J}$ a $1600 \mathrm{~K}$ planet. The limits rapidly become less stringent for cooler planets. The above limits are based on a model in which the depth of the $\mathrm{K}$ band water band is $69 \%$. If the water absorption is deeper or shallower this affects the sensitivity of the technique correspondingly. In the $\mathrm{T}$ dwarf Gl229b the depth of absorption is approximately $80 \%$. However in earlier $\mathrm{T}$ dwarfs (Leggett et al. 2000 ), which may have $\mathrm{T}_{\text {eff }} \approx 1600 \mathrm{~K}$, the absorption depth is only $45 \%$.

\section{References}

Charbonneau, Brown, Latham \& Mayor 1999, ApJ, 529, L45

Henry, Marcy, Butler \& Vogt 2000, ApJ, 529, L41

Leggett, Geballe, Fan + SLOAN collaboration 2000, ApJ, 536, L35 